\title{
The irrationality of some number theoretical series
}

\author{
by \\ J.-C. Schlage-Puchta (Freiburg)
}

In this note we prove the irrationality of some series by combining methods from elementary and analytic number theory with methods from the theory of uniform distribution. Our first result yields an explicit set of uncountably many $\mathbb{Q}$-linearly independent real numbers.

Theorem 1. For a real number $\lambda \geq 0$ define the series

$$
S_{\lambda}=\sum_{n \geq 0} \frac{\left[n^{\lambda}\right]}{n !} .
$$

Then the set $\{1, e\} \cup\left\{S_{\lambda}: \lambda \in(0, \infty) \backslash \mathbb{Z}\right\}$ is $\mathbb{Q}$-linearly independent.

Some properties of the function $S_{\lambda}$ are given in the following.

Proposition 1. The function $\lambda \mapsto S_{\lambda}$ is injective, monotone, and continuous from the right. Its image has cardinality of the continuum, Hausdorff dimension 0 , and it is totally disconnected.

Our second theorem deals with real numbers defined by their digital expansion.

TheORem 2. Let $b \geq 2$ be an integer, not a proper power. Let $g: \mathbb{N} \rightarrow \mathbb{R}$ be a continuous non-decreasing function such that $g(n+1) / g(n) \rightarrow 1$. Let $f: \mathbb{N} \rightarrow \mathbb{N}$ be a non-decreasing function such that $f(n+1) / f(n) \sim g(n)$, and denote by $\alpha$ the real number with base $b$ expansion $0 . f(1) f(2) f(3) \ldots$ Suppose that $\alpha$ is rational. Then $g$ converges to some constant $c$ as $n \rightarrow \infty$, $c$ is a power of $b$, and $f(n+1)=c f(n)+\mathcal{O}(1)$.

Note that there do exist functions $f, g$ such that $\alpha$ is rational, for example, taking $b=10, f(n)=\left(10^{n}-1\right) / 9$, and $g(n) \rightarrow 10$ we find $\alpha=1 / 9$. For the function $f(n)=a^{n}, a \in \mathbb{N}, a \geq 2$, this result was proven for base $b=10$ by Mahler [7] and for arbitrary $b$, including the case of $b$ being a proper power, by Bundschuh [1].

2000 Mathematics Subject Classification: Primary 11J72. 
Denote by $p_{n}$ the $n$th prime number, and, for an integer $k \geq 0$, define the series $S_{k}$ by

$$
S_{k}:=\sum_{n=1}^{\infty} \frac{p_{n}^{k}}{n !}
$$

P. Erdös [2] stated that $S_{k}$ is irrational and gave a proof for $k=1$. However, it appears that, for $k>1$, no proof has appeared in print. Our last result is the following.

TheOREM 3. The real numbers $1, S_{0}, S_{1}, S_{2}, \ldots$ are $\mathbb{Q}$-linearly independent.

Our proofs will use the following results from the theory of equidistribution (cf., for example, [3, Theorem 2.8] and [6, II, Theorem 2.5]).

Lemma 1 (Weyl-van der Corput). Let $f$ be a function which is $(q+2)$ times continuously differentiable, and suppose that $\lambda \leq f^{(q+2)}(t) \leq \alpha \lambda$ on the interval $[1, N]$. Then for $q=0$ we have

$$
\sum_{n=1}^{N} e(f(n)) \ll \alpha N \lambda^{1 / 2}+\lambda^{-1 / 2}
$$

whereas for $q \geq 1$ we have

$$
\sum_{n=1}^{N} e(f(n)) \ll N\left(\alpha^{2} \lambda\right)^{1 /(4 Q-2)}+N^{1-1 / 2 Q} \alpha^{1 / 2 Q}+N^{1-1 / 2 Q+1 / Q^{2}} \lambda^{-1 / 2 Q},
$$

where $Q=2^{q}$.

LEMma 2 (Erdős-Turán). Let $\left(x_{n}\right)_{n=1}^{N}$ be a sequence of real numbers in the interval $[0,1]$. Then the discrepancy $D_{N}$ of this sequence is bounded above by

$$
D_{N} \ll \frac{N}{H}+\sum_{1 \leq h \leq H}\left|\sum_{n=1}^{N} e\left(h x_{n}\right)\right| .
$$

Proof of Theorem 1. It suffices to show that for all tuples $a_{1}, \ldots, a_{k} \in$ $\mathbb{Z} \backslash\{0\}$ and $0 \leq \lambda_{1}<\cdots<\lambda_{k}$ such that no $\lambda_{i}$ is an integer $\geq 2$, the real number

$$
S=\sum_{n \geq 0} \frac{1}{n !}\left(a_{1}\left[n^{\lambda_{1}}\right]+\cdots+a_{k}\left[n^{\lambda_{k}}\right]\right)
$$

is irrational. Moreover, we may assume that at least one of the $\lambda_{i}$ is not 0 . Suppose that $S=p / q$, and let $n \geq q$ be an integer. Then $n ! \cdot S \in \mathbb{Z}$, and we deduce

$$
\left\|\sum_{\nu \geq 1} \frac{1}{(n+1) \cdots(n+\nu)}\left(a_{1}\left[(n+\nu)^{\lambda_{1}}\right]+\cdots+a_{k}\left[(n+\nu)^{\lambda_{k}}\right]\right)\right\|=0
$$


where $\|\cdot\|$ denotes the distance to the nearest integer. Set $M=\left[\lambda_{k}\right]+1$. Then truncating the series at $\nu=M$ yields an error of size $\mathcal{O}\left(n^{-1}\right)$. Neglecting the rounding introduces an error of the same magnitude, and we obtain

$$
\left\|\sum_{\nu=1}^{M} \frac{1}{(n+1) \cdots(n+\nu)}\left(a_{1}(n+\nu)^{\lambda_{1}}+\cdots+a_{k}(n+\nu)^{\lambda_{k}}\right)\right\| \ll \frac{1}{n} .
$$

If $\lambda_{k}<1$, the sum collapses to a single term, which tends to 0 , and we obtain $n^{\lambda_{k}-1} \ll n^{-1}$, contradicting the assumption that at least one of the $\lambda_{i}$ is non-zero. If $\lambda_{k}>1$, define

$$
f(t)=\sum_{\nu=1}^{M} \frac{1}{(t+1) \cdots(t+\nu)}\left(a_{1}(t+\nu)^{\lambda_{1}}+\cdots+a_{k}(t+\nu)^{\lambda_{k}}\right) .
$$

For $t>M, f$ is analytic, and, since $\lambda_{k} \notin \mathbb{Z}$, there exist some $K \in \mathbb{N}$ such that $f^{(K+1)}(t)$ and $f^{(K+2)}(t)$ do not change sign for $t>t_{0}$, and

$$
\lim _{t \rightarrow \infty} \frac{f^{(K)}(t)}{t}=0, \quad \lim _{t \rightarrow \infty} \frac{1}{t f^{(K+1)}(t)}=0 .
$$

Lemma 1 now implies that the sequence $f(n)$ is equidistributed modulo 1 (cf., e.g., [5, pp. 36-39]). However, the latter statement clearly contradicts (1), which proves our theorem.

Proof of Proposition 1. Monotonicity is clear. Suppose that $\lambda_{2}>\lambda_{1}$. Then for $n \geq n_{0}$ we have $n^{\lambda_{2}}>n^{\lambda_{1}}+1$, and therefore $\left[n^{\lambda_{2}}\right] \geq\left[n^{\lambda_{1}}\right]+1$, which implies $S_{\lambda_{2}} \geq S_{\lambda_{1}}+1 / n_{0}$ !, and we conclude that the map $\lambda \mapsto S_{\lambda}$ is injective. Hence, the image of this map has the same cardinality as its domain, which is the continuum. We now prove continuity from the right. For each $n$ there is some $\varepsilon_{n}$ such that $\left[n^{\lambda}\right]=\left[n^{t}\right]$ for all $t \in[\lambda, \lambda+\varepsilon]$. Let $N$ be a sufficiently large integer, and set $\varepsilon=\min \left(1,\left\{\varepsilon_{n}: n \leq N\right\}\right)$. Then for $t \in[\lambda, \lambda+\varepsilon]$ we have the bound

$$
S_{t}-S_{\lambda}=\sum_{n=1}^{\infty} \frac{\left[n^{t}\right]-\left[n^{\lambda}\right]}{n !}=\sum_{n=N+1}^{\infty} \frac{\left[n^{t}\right]-\left[n^{\lambda}\right]}{n !} \leq \sum_{n=N+1}^{\infty} \frac{n^{\lambda+1}}{n !} \leq 2 \frac{N^{\lambda}}{N !},
$$

which tends to 0 as $N \rightarrow \infty$. Hence, if $\lambda_{i} \searrow \lambda$, then $S_{\lambda_{i}} \searrow S_{\lambda}$, and $S_{\lambda}$ is continuous from the right. The fact that the image of $S_{\lambda}$ is totally disconnected follows from Theorem 1 , since a connected component would contain some interval of positive length, and therefore infinitely many rational numbers, only finitely many of which are excluded by the condition $\lambda \notin \mathbb{Z}$.

To estimate the Hausdorff dimension, let $N$ be an integer, and define $S_{\lambda}^{N}$ as the partial sum $\sum_{n=1}^{N}\left[n^{\lambda}\right] / n !$. The image $\mathcal{I}_{N}$ of an interval $[t, t+1]$ under the map $\lambda \mapsto S_{\lambda}^{N}$ consists of finitely many points, more precisely, the cardinality of the image of $[t, t+1]$ is at most the number of values $\lambda \in[t, t+1]$ such that $n^{\lambda}$ is integral for some $n \leq N$, and this quantity is 
bounded above by $N^{t+2}$. Clearly, $S_{\lambda}^{N} \leq S_{\lambda}$, and for $\lambda \in[t, t+1]$ we have, for every fixed $A>0$ and $N$ sufficiently large, the estimate

$$
\left|S_{\lambda}^{N}-S_{\lambda}\right|=\sum_{n>N} \frac{\left[n^{\lambda}\right]}{n !}<\sum_{n>N} \frac{n^{t+1}}{n !}<\frac{N^{t+1}}{N !}<N^{-A} .
$$

Hence, for $N$ large the image $\mathcal{I}$ of $[t, t+1]$ under the map $\lambda \mapsto S_{\lambda}$ can be convered by $N^{t+2}$ intervals of length $N^{-A}$ each, thus, the Hausdorff dimension of $\mathcal{I}$ is bounded above by $(t+2) / A$ for any $A$, and therefore the Hausdorff dimension is 0 .

Proof of Theorem 2. For a positive integer $n$, denote by $\ell(n)$ the number of digits of $n$, and by $\log n$ the logarithm in base $b$. Suppose that $\alpha$ is rational. Then the sequence of digits of $\alpha$ is ultimately periodic with period $p$, say.

There are only $p$ cyclic permutations of the digits of the period of $\alpha$, hence, $\log f(n) \bmod 1$ has at most $p$ limit points; order these limit points as $0 \leq x_{1}<\cdots<x_{m}<1$. In particular, for every $\varepsilon>0$ there exists some $n_{0}$ such that for $n>n_{0}$ we have $\left(f(n)-x_{i}\right) \bmod 1<\varepsilon$ for some $i$ depending on $n$. Moreover, increasing $n_{0}$ if necessary, we may assume that $|\log f(n+1)-\log f(n)-\log g(n)|<\varepsilon$, and obtain

$\log g(n) \bmod 1=(\log f(n+1)-\log f(n)) \bmod 1+\delta_{1}=x_{i}-x_{j}+\delta_{2}$ for some indices $i, j$, and real numbers $0 \leq \delta_{1}, \delta_{2}<\varepsilon$. Hence, $\log g(n) \bmod 1$ has finitely many limit points as well. However, since $\log g(n+1)-\log g(n)$ $\rightarrow 0$, this implies that $\log g(n)$ converges, thus, $g(n) \rightarrow c$ for some constant $c$.

We now distinguish two cases, depending on whether $\log c / \log b$ is rational or not.

Suppose that $\log c / \log b$ is rational. Since $f(n+1) / f(n)$ converges, all but finitely many $n$ have the property that there are infinitely many $m$ such that $\ell(f(n)) \equiv \ell(f(m)) \bmod p$, and that $f(n)$ and $f(m)$ begin with the same $p$ digits. This implies in particular that $f(n+1)$ and $f(m+1)$ begin with the same $p$ digits. Since $f(m+1) / f(m) \rightarrow c$, and there are infinitely many $m$ at our disposal, we may choose $m$ so large that

$$
\left|\frac{f(m+1)}{f(m)}-c\right|<\frac{1}{f(n)}
$$

while periodicity implies

$$
\left|\frac{f(m+1)}{f(m)}-\frac{f(n+1)}{f(n)}\right|<\frac{1}{f(n)},
$$

hence, $f(n+1)=c f(n)+\mathcal{O}(1)$ holds true for all $n$. Furthermore, for a sequence $n_{i}$ such that $\ell\left(f\left(n_{i}\right)\right) \bmod p$ and the first $p$ digits of $f\left(n_{i}\right)$ are constant, the rational numbers $\beta$ and $\gamma$ obtained from $\alpha$ by shifting the decimal point right in front of the first digit belonging to $f\left(n_{i}\right)$ resp. $f\left(n_{i}+1\right)$ 
do not depend on $i$. Hence,

$$
c=\lim _{i \rightarrow \infty} \frac{f\left(n_{i}+1\right)}{f\left(n_{i}\right)}=\lim _{i \rightarrow \infty} \frac{\beta b^{\ell\left(f\left(n_{i}+1\right)\right)}+\mathcal{O}(1)}{\gamma b^{\ell\left(f\left(n_{i}\right)\right)}+\mathcal{O}(1)}=\frac{\beta b^{\ell\left(f\left(n_{i}\right)+1\right)-\ell\left(f\left(n_{i}\right)+1\right)}}{\gamma}
$$

is rational. Hence, $c$ is both rational and a rational power of $b$, which implies that either $b$ is a proper power, or $c$ is a proper power of $b$. Hence, our theorem holds true in this case.

Now we suppose that $\log c / \log b$ is irrational; we have to show that this assumption leads to a contradiction. For any irrational number $\alpha$ the sequence $(\alpha n / p)_{n=1}^{\infty}$ is equidistributed modulo 1, in particular, there are infinitely many $n$ such that $\ell(f(n))$ is divisible by $p$ and $f(n)$ does not have both its leading digits equal to $b-1$. For such $n, f(n)$ and $f(n+1)$ begin with the same digits, in fact, $f(n)$ is an initial segment of $f(n+1)$. Hence, $f(n+1)=f(n) \cdot b^{k}+\mathcal{O}\left(b^{k}\right)$ for some positive integer $k$. Since $g(n) \rightarrow c$, we deduce that $c$ itself is a power of $b$, contradicting the assumption that $\log c / \log b$ is irrational, and the theorem is proven.

For the proof of Theorem 3 we need some auxiliary results. The first result is a consequence of Selberg's sieve (cf., e.g., [4, Theorem 5.1]).

Lemma 3. Let $0 \leq a_{1}<\cdots<a_{k}<N$ be a sequence of integers, and let $\mathcal{N} \subseteq[x, 2 x]$ be a set of integers such that for all $n \in \mathcal{N}$ each of the integers $n+a_{i}$ is prime. Then

$$
|\mathcal{N}| \leq \frac{c_{k} x}{\log ^{k+1} x} \prod_{p}\left(1+\frac{1}{p}\right)^{k+2-\nu(p)},
$$

where $\nu(p)$ denotes the number of distinct residues $\bmod p$ among

$$
\left\{0, \Delta_{0}, \Delta_{0}+\Delta_{1}, \ldots, \Delta_{0}+\cdots+\Delta_{k}\right\} \text {. }
$$

In particular (with $\log _{2}$ denoting the iterated logarithm),

$$
|\mathcal{N}| \ll_{k} \frac{x \log _{2}^{k+2} x}{\log ^{k+1} x}
$$

For the rest of this article, set $\delta_{n}=p_{n+1}-p_{n}$.

Lemma 4. Let $F \in \mathbb{Z}\left[x_{0}, \ldots, x_{k}\right]$ be a polynomial which does not vanish identically. Then $F\left(\delta_{n}, \ldots, \delta_{n+k}\right) \neq 0$ for almost all $n$.

Proof. Neglecting $\mathcal{O}\left(x / \log _{2} x\right)$ indices at most, we may assume that $\delta_{i} \leq$ $\log x \log \log x$ for $n \leq i \leq n+k$. For a fixed tuple $\Delta_{0}, \ldots, \Delta_{k}$ satisfying $\Delta_{i} \leq \log x \log _{2} x$, the number of solutions $n$ of the equations $\delta_{n+i}=\Delta_{i}$, $0 \leq i \leq k$, is bounded above by the number $N$ of primes $p \in\left[p_{x}, p_{2 x}\right]$ with the property that $p+\Delta_{0}+\cdots+\Delta_{i}$ is prime for all $0 \leq i \leq k$, and from Lemma 3 we infer that this quantity is $\mathcal{O}\left(x \log _{2}^{k+2} x / \log ^{k+1} x\right)$. Since $F$ does not vanish identically, the number of tuples $\left(\Delta_{0}, \ldots, \Delta_{k}\right)$ such that 
$F\left(\Delta_{0}, \ldots, \Delta_{k}\right)=0$ and $\Delta_{i} \leq \log x \log _{2} x$ for all $i$, is of size $\mathcal{O}\left(\log ^{k} x \log _{2}^{k} x\right)$, hence, the total number of solutions of the equation $F\left(\delta_{n}, \ldots, \delta_{n+k}\right)=0$ is $\ll x \log _{2}^{2 k+2} x / \log x$, which is sufficiently small.

Lemma 5. Let $k$ be a field, $P, Q \in k\left[X_{1}, \ldots, X_{n}\right]$ be polynomials, and $\nu \neq 0$ an integer such that

$$
\begin{aligned}
\nu X_{1} P\left(X_{1}, \ldots, X_{n}\right)+P\left(X_{1}, \ldots, X_{n}\right) & Q\left(X_{2}, \ldots, X_{n+1}\right) \\
& -P\left(X_{2}, \ldots, X_{n+1}\right) Q\left(X_{1}, \ldots, X_{n}\right)
\end{aligned}
$$

vanishes identically. Then $P$ vanishes identically.

Proof. Suppose $Q \neq 0$, and put $X_{1}=0$. Then the polynomial

$$
P\left(0, X_{2}, \ldots, X_{n}\right) Q\left(X_{2}, \ldots, X_{n+1}\right)-P\left(X_{2}, \ldots, X_{n+1}\right) Q\left(0, X_{2}, \ldots, X_{n}\right)
$$

vanishes identically, that is, putting $R\left(X_{1}, \ldots, X_{n}\right)=\frac{P\left(X_{1}, \ldots, X_{n}\right)}{Q\left(X_{1}, \ldots, X_{n}\right)}$ we find that

$$
R\left(0, X_{2}, \ldots, X_{n}\right)=R\left(X_{2}, \ldots, X_{n+1}\right)
$$

holds identically. In particular, $R\left(X_{1}, \ldots, X_{n}\right)$ does not involve $X_{n}$ at all. Hence,

$$
R\left(0, X_{2}, \ldots, X_{n-1}, 0\right)=R\left(X_{2}, \ldots, X_{n}, 0\right)
$$

holds identically, and we deduce that $R$ does not involve $X_{n-1}$ either. Continuing in this way we infer that $R$ is constant, that is, the polynomial

$$
P\left(X_{1}, \ldots, X_{n}\right) Q\left(X_{2}, \ldots, X_{n+1}\right)-P\left(X_{2}, \ldots, X_{n+1}\right) Q\left(X_{1}, \ldots, X_{n}\right)
$$

and therefore also $\nu X_{1} P\left(X_{1}, \ldots, X_{n}\right)$ vanishes identically. However, since $\nu \neq 0$, this can only happen if $P$ vanishes.

Lemma 6. Let $Q \in \mathbb{Z}[X]$ be a non-constant polynomial of degree $d$ and coefficients bounded by $M$. Then the discrepancy $D$ of the sequence $\left(Q\left(p_{n} / n\right) \bmod 1\right)_{n=x}^{2 x}$ is bounded above by

$$
D \ll x e^{-c \sqrt{\log x}}+M^{1 / 3} x^{2 / 3} \log ^{d / 3} x .
$$

Proof. For $t \geq 2$, denote by $f(t)$ the inverse function of lit, that is, the unique positive solution of the equation

$$
\int_{2}^{f(t)} \frac{d x}{\log x}=t .
$$

Then, by the prime number theorem, we deduce that

$$
Q\left(\frac{p_{n}}{n}\right)-Q\left(\frac{f(n)}{n}\right) \ll e^{-c \sqrt{\log n}},
$$

hence, it suffices to estimate the discrepancy $\widetilde{D}$ of the sequence

$$
\left(Q\left(f(n) n^{-1}\right) \bmod 1\right)_{n=x}^{2 x} .
$$


We will do so using Lemma 2. Set $F(t)=Q\left(f(t) t^{-1}\right)$. Then for every integer $H \geq 1$ we have

$$
\widetilde{D} \ll \frac{x}{H}+\frac{1}{h} \sum_{h \leq H}\left|\sum_{n=x}^{2 x} e(h F(n))\right| .
$$

The second derivative of $h F$ is of size $h t^{-1} M \log ^{d} t$. We can now apply the case $q=0$ of Lemma 1 to deduce

$$
\sum_{n=x}^{2 x} e(h F(n)) \ll M^{1 / 2} x^{1 / 2} h^{1 / 2} \log ^{d / 2} x,
$$

and therefore

$$
\widetilde{D} \ll \frac{x}{H}+M^{1 / 2} x^{1 / 2} H^{1 / 2} \log ^{d / 2} x \ll M^{1 / 3} x^{2 / 3} \log ^{d / 3} x .
$$

Proof of Theorem 3. It suffices to show that

$$
S=\sum_{\nu=1}^{\infty} \frac{P\left(p_{\nu}\right)}{\nu !}
$$

is irrational for every polynomial $P$ with integral coefficients which does not vanish identically. Assume to the contrary that $S$ is rational. Then, for $n$ sufficiently large, $n ! S$ is an integer, and we deduce that

$$
\sum_{\nu=n+1}^{\infty} \frac{P\left(p_{\nu}\right)}{(n+1) \cdots \nu}
$$

is an integer. Denote the degree of $P$ by $k$. Since $p_{\nu} \sim \nu \log \nu$, we deduce

$$
\left\|\sum_{\nu=1}^{k-1} \frac{P\left(p_{n+\nu}\right)}{(n+1) \cdots(n+\nu)}\right\| \ll \frac{\log ^{k} n}{n}
$$

for all $n$ sufficiently large. Set

$$
F^{(0)}(n)=\sum_{\nu=1}^{k-1} \frac{P\left(p_{n+\nu}\right)}{(n+1) \cdots(n+\nu)} .
$$

In the following we shall write $R(n)$ to denote a negligible error term, that is, any function satisfying $R(n) \ll\left(\log ^{c} n\right) / n$ for almost all $n$ and some constant $c$. In particular, we have the relation $\delta_{n} R(n)=R(n)$. Expanding $1 /(n+1) \cdots(n+k)$ in a Laurent series around 0 , and expressing $p_{n+k}$ by $p_{n}$ and the $\delta_{n+i}$ 's, we find

$$
F^{(0)}(n)=\sum_{\nu=1}^{k} \sum_{\mu=1}^{\nu} P_{\nu \mu}^{(0)}\left(\delta_{n}, \ldots, \delta_{n+k-1}\right) \frac{p_{n}^{\nu}}{n^{\mu}}+R(n) .
$$

Define a partial order on the set of pairs $(\nu, \mu)$ by $\left(\nu_{1}, \mu_{1}\right) \succ\left(\nu_{2}, \mu_{2}\right)$ if $\nu_{1}-\mu_{1}>\nu_{2}-\mu_{2}$, or $\nu_{1}-\mu_{1}=\nu_{2}-\mu_{2}$ and $\nu_{1}>\nu_{2}$; that is, the corresponding 
fraction $p_{n}^{\nu_{1}} / n^{\mu_{1}}$ has faster growth than $p_{n}^{\nu_{2}} / n^{\mu_{2}}$. We now define a sequence of functions $F^{(i)}$ and $P_{\mu \nu}^{(i)}$ recursively by

$F^{(i+1)}(n)=P_{\nu_{0} \mu_{0}}^{(i)}\left(\delta_{n+1}, \ldots, \delta_{n+k}\right) F^{(i)}(n)-P_{\nu_{0} \mu_{0}}^{(i)}\left(\delta_{n}, \ldots, \delta_{n+k-1}\right) F^{(i)}(n+1)$

where $\left(\nu_{0}, \mu_{0}\right)$ is maximal with respect to $\succ$ among all pairs with the property that $P_{\nu \mu}^{(i)}$ is non-trivial, and

$$
F^{(i)}(n)=\sum_{\nu=1}^{k-1} \sum_{\mu=1}^{\nu} P_{\nu \mu}^{(i)}\left(\delta_{n}, \ldots, \delta_{n+k}\right) \frac{p_{n}^{\nu}}{n^{\mu}}+R(n) .
$$

We have

$$
\begin{aligned}
P_{\nu_{0}-1, \mu_{0}}^{(i+1)}= & \nu_{0} P_{\nu_{0} \mu_{0}}^{(i)}\left(\delta_{n}, \ldots, \delta_{n+k-1}\right) \delta_{n} \\
& +P_{\nu_{0} \mu_{0}}^{(i)}\left(\delta_{n+1}, \ldots, \delta_{n+k}\right) P_{\nu_{0}-1, \mu_{0}}^{(i)}\left(\delta_{n}, \ldots, \delta_{n+k-1}\right) \\
& -P_{\nu_{0} \mu_{0}}^{(i)}\left(\delta_{n}, \ldots, \delta_{n+k-1}\right) P_{\nu_{0}-1, \mu_{0}}^{(i)}\left(\delta_{n+1}, \ldots, \delta_{n+k}\right) .
\end{aligned}
$$

Since $\nu_{0} \geq 1$, we deduce from Lemma 5 that $P_{\nu_{0}-1, \mu_{0}}^{(i+1)}$ does not vanish identically. In each step, the pair $\left(\nu_{0}, \mu_{0}\right)$ is removed from the set of pairs occurring in $F^{(i)}$, and all pairs $(\nu, \mu)$ added satisfy $\nu-\mu<\nu_{0}-\mu_{0}$; thus, after finitely many steps the maximum of $\nu-\mu$ is reduced by 1 , and again after finitely many steps the maximum is reduced to 0 , that is, we reach some $F^{(i)}$ such that $\mu=\nu$ for all $(\mu, \nu)$ with $P_{\mu \nu} \neq 0$, and with at least one pair $(\mu, \nu)$ such that $P_{\mu \nu} \neq 0$. Moreover, we have $\left\|F^{(i)}(n)\right\|=R(n)$. During the recursive process leading to $F^{(i)}$, we multiplied the initial polynomial, which had rational coefficients, with other polynomials with rational coefficients, and shifted indices. Hence, there exist some integer $\ell$ and polynomials $Q_{i} \in \mathbb{Q}\left[X_{1}, \ldots, X_{\ell}\right], 1 \leq i \leq \ell, Q_{\ell} \neq 0$, such that

$$
\left\|\sum_{i=1}^{\ell} Q_{i}\left(\delta_{n}, \ldots, \delta_{n+\ell}\right) \frac{p_{n}^{i}}{n^{i}}\right\|=R(n) .
$$

Moreover, since $c \cdot R(n)=R(n)$ for every constant $c$, we may multiply with all occurring denominators, and suppose that each $Q_{i}$ has integral coefficients. Therefore, we can apply Lemma 4 and find that for almost all $n$, one of the polynomials $Q_{i}\left(\delta_{n}, \ldots, \delta_{n+\ell}\right)$ does not vanish, while at the same time, for almost all $n$ none of the differences $\delta_{n}, \ldots, \delta_{n+\ell}$ exceeds $\log ^{2} n$. Hence, setting $a_{i}=Q_{i}\left(\delta_{n}, \ldots, \delta_{n+\ell}\right)$, we find that for almost all $n$ there are integers $a_{1}, \ldots, a_{\ell}$, not all zero, with $0<\left|a_{i}\right|<\log ^{A} n$ for some constant $A$ depending only on the initial polynomial $P$, such that

$$
\left\|\sum_{i=1}^{\ell} a_{i} \frac{p_{n}^{i}}{n^{i}}\right\| \ll e^{-c \sqrt{\log n}} .
$$

In particular, there are integers $a_{1}, \ldots, a_{\ell}$ such that $(2)$ is satisfied for at 
least $x / \log ^{\ell A} x$ integers $n \leq x$ for some constant $A$. This clearly contradicts Lemma 6, thereby proving Theorem 3.

\section{References}

[1] P. Bundschuh, Generalization of a recent irrationality result of Mahler, J. Number Theory 19 (1984), 248-253.

[2] P. Erdős, Sur certaines séries à valeur irrationnelle, Enseign. Math. (2) 4 (1958), 93100.

[3] S. W. Graham and G. Kolesnik, van der Corput's Method of Exponential Sums, London Math. Soc. Lecture Note Ser. 126, Cambridge Univ. Press, Cambridge, 1991.

[4] H. Halberstam and H.-E. Richert, Sieve Methods, London Math. Soc. Monogr. 4, Academic Press, London, 1974.

[5] E. Hlawka, Theorie der Gleichverteilung, Bibliographisches Institut, Mannheim, 1979.

[6] L. Kuipers and H. Niederreiter, Uniform Distribution of Sequences, Pure Appl. Math., Wiley-Interscience, New York, 1974.

[7] K. Mahler, On some irrational decimal fractions, J. Number Theory 13 (1981), 268269.

Mathematisches Institut

Eckerstr. 1

79104 Freiburg, Germany

E-mail: jcsp@mathematik.uni-freiburg.de

Received on 13.12.2004

and in revised form on 20.11.2006 